\title{
El contexto vivo: reflexiones sobre una experiencia etnográfica colaborativa universitaria
}

The Living Context: Reflections about a Collaborative University Ethnographic Experience

Artículo de reflexión | Reflexion article

Fecha de recepción: 15 de julio de 2019 Fecha de aceptación: 04 de octubre de 2019 Fecha de disponibilidad en línea: julio de 2020

doi: 10.11144/Javeriana.m14.cvre

Vilma Huerta-Cordova v_huerta@yahoo.com Universidad Autónoma Benito Juárez de Oaxaca, México (D) ORCID: https://orcid.org/0000-0002-2914-7132

Ángeles Clemente-Olmos angelesclementeo@gmail.com Universidad Autónoma Benito Juárez de Oaxaca, México (D) ORCID: https://orcid.org/0000-0002-7511-8914

Lorena Córdova-Hernández Icordova.cat@uabjo.mx

Universidad Autónoma Benito Juárezz de OAxaca, México (D) ORCID: https://orcid.org/0000-0002-2681-7102

Para citar este artículo | To cite this article Huerta-Cordova, V., Clemente-Olmos, A. \& Córdova-Hernández, L. (2021). El contexto vivo: reflexiones sobre una experiencia etnográfica colaborativa universitaria. magis, Revista Internacional de Investigación en Educación, 14, 1-20. doi: 10.11144/Javeriana.m14.cvre 


\title{
Resumen
}

Se describe un ejercicio etnográfico colaborativo desarrollado por universitarios futuros docentes de idiomas para la observación y descripción del contexto vivo de su investigación, cuyo producto final es un texto académico en coautoría que forma parte de su tesis de grado. En específico, este artículo analiza cómo los estudiantes se posicionaron como investigadores y, a partir de ello, cómo describieron las condiciones de su espacio de observación y visibilizaron a los actores sociales. Asimismo, se analizan sus testimonios sobre el aprendizaje adquirido en el momento de organizar la observación etnográfica, así como la negociación de los conflictos surgidos durante la colaboración.

\section{Palabras clave}

Educación; estudiante; etnografía; investigación participativa

\begin{abstract}
It describes a collaborative ethnographic exercise developed by university students, future language teachers; for the observation and description of the "living context" of their research whose final product is a co-authored academic text which makes part of their graduation project. Specifically, the article analyses how students became researchers and, from there, how they described the conditions of their observation context and made social actors visible. Likewise, their testimonials about the acquired knowledge when organizing ethnographic observation are analyzed as well as the negotiation of conflicts which emerged during collaboration.
\end{abstract}

\section{Keywords}

Education; students; ethnography; participatory research 
Descripción del artículo | Article description

Artículo de reflexión derivado de la investigación Titulación, investigación y conciencia pedagógica.

\section{Introducción}

El presente artículo es una reflexión sobre el proceso de investigación llevado a cabo por 30 estudiantes universitarios que cursaban la Licenciatura en Enseñanza de Idiomas en la Universidad Autónoma Benito Juárez de Oaxaca (UABJO) para realizar trabajo de campo en su propio espacio escolar, con el objetivo de conformar el capítulo referente al contexto de la investigación en el marco de su proyecto de titulación. Para ello, los estudiantes emplearon la colaboración etnográfica, la cual se considera aquí como "una forma de proceder y construir conocimiento de una manera compartida" (Rappaport, 2007, p. 6). El producto derivado de la investigación etnográfica fue un texto académico en coautoría, denominado contexto vivo. Dicho contexto es una metáfora que utilizó uno de los estudiantes para hacer referencia al contexto real o contexto vivido de la interacción social.

A partir de lo anterior, la reflexión aquí presentada se basa en la narrativa etnográfica de una de las autoras como docente del curso Proyecto de Investigación, de la Licenciatura en la Enseñanza de Idiomas. Como se mencionó, estos 30 estudiantes realizaron dicho proyecto en el mismo espacio en el que estudian e interactúan, pero sobre el que normalmente no se habla o se describe en las tesis de grado. Por tal motivo, el desarrollo de la etnografía se consideró pertinente para favorecer la puesta en marcha de la etnografía colaborativa, pues "facilita la construcción del conocimiento con el 'otro' y no sobre el 'otro'" (Guerrero, 2018, p. 36). Sin embargo, antes de introducir al lector en la narrativa etnográfica, es necesario hacer algunas aclaraciones sobre cómo surge la propuesta de trabajo con los estudiantes.

Dentro del programa de licenciatura, el proceso de titulación inicia en el quinto semestre y concluye en el octavo, con la defensa de la tesis ante un jurado. Las materias orientadas a desarrollar la tesis o trabajo de titulación son cuatro, y todas ellas son impartidas por un mismo docente, quien evalúa los avances al término de cada semestre. Las tesis elaboradas por los alumnos están estructuradas en cuatro capítulos. Uno de ellos desarrolla el contexto de la investigación, cuyo objetivo es describir las diferentes características que conforman el espacio y el momento en 
donde se lleva a cabo la indagación. Antes de esta propuesta, una de las maneras de abordar el capítulo del contexto de la investigación era a través de la consulta de documentos oficiales, por ejemplo, la página web y el Plan Institucional de Desarrollo de la Facultad. Sin embargo, en este caso, las autoras discutimos sobre la necesidad de dar un giro a esta situación, por medio de dos estrategias: 1) desarrollar la descripción del contexto de estudio desde un enfoque etnográfico y 2) propiciar la colaboración explícita en este proceso etnográfico para la construcción del contexto de la investigación.

Los fundamentos en los que las autoras nos basamos para trabajar la etnografía con los estudiantes fueron tres. El primero fue tomar en cuenta que los estudiantes, durante sus estudios de licenciatura, se han formado como profesores de lenguas a través de una larga tradición en etnografía educativa (Canagarajah, 1999; Heath, 1984; Woods, 1993), la cual alienta a los estudiantes a llevar a cabo sus propias etnografías para observar, entender y analizar la cultura de la lengua que están aprendiendo (Roberts, Byram, Barro, Jordan \& Street, 2001).

Dentro de la tradición de investigación social de este programa, también se han desarrollado estudios etnográficos en la propia escuela de los estudiantes que formaron parte de esta investigación sobre el contexto vivo, entre los cuales podemos encontrar el efectuado por Clemente \& Higgins (2008) y, más recientemente, el realizado por López-Gopar (2016), en los que se buscó que los estudiantes desarrollaran diferentes habilidades etnográficas, como la elaboración de sus autobiografías en forma de retratos etnográficos.

El segundo motivo fue el hecho de que la etnografía, tomada no solamente como metodología cualitativa, sino como una posición en la investigación social —epistemológica, metodológica y escritural (Milstein, Clemente, Dantas-Whitney, Guerrero \& Higgins, 2011)—, daría a los estudiantes las habilidades necesarias para adentrarse en su propio contexto universitario, a través de la recolección e interpretación de las prácticas sociales y la manera en que estas actividades se reproducen en el contexto sociocultural de su cotidianidad como estudiantes (Geertz, 1973). De esta manera, se consideró propicio compartir el mismo contexto de interacción de los estudiantes y la docente para el proceso de colaboración, lo cual, a su vez, permitió entablar una mejor orientación en la investigación.

El tercer fundamento fue privilegiar el trabajo colaborativo para propiciar que los estudiantes coprodujeran a nivel metodológico, interpretativo y de redacción el producto final, pues este proceso no solo consiste en trabajar de manera colectiva, sino, además, en "aprender a respetar el conocimiento y habilidades que cada miembro aporta a la discusión con 
el fin de desarrollar proyectos de investigación" (Domínguez-Gaona, Crhová \& Molina-Landeros, 2015).

Hoy en día se ha puntualizado la necesidad de explicitar la colaboración dentro de los diferentes aspectos del proceso etnográfico (Milstein et al., 2011). Así pues, podemos encontrar diversos grados de colaboración etnográfica, desde la cointerpretación entre investigador y participantes, pasando por la cogeneración de conocimiento, hasta llegar a la coinvestigación y la coautoría (Huerta-Cordova, Clemente \& Córdova-Hernández, 2019). Todos estos grados se refieren a colaboración de tipo vertical, es decir, entre los investigadores y sus participantes. Sin embargo, la naturaleza de la colaboración que se presenta en este estudio es horizontal, pues se refiere exclusivamente a la colaboración entre los estudiantes (que se inician en la investigación etnográfica) para realizar el estudio del contexto vivo. No obstante, es importante señalar que la horizontalidad se dio solamente para el trabajo coinvestigativo sobre la observación y descripción del contexto, pues existió una verticalidad constante en la relación estudiante-docente durante todo el proceso de investigación, en términos de determinar los objetivos, metas, temporalidades, etc., del proceso. Además de lo señalado, el análisis presentado en este artículo es una clara representación de una relación vertical, pues en el análisis y la reflexión no participaron los estudiantes.

\section{La etnografía: la construcción del contexto vivo}

Para desarrollar el trabajo etnográfico con los estudiantes universitarios se tomó el modelo propuesto por Podestá-Siri (Niños y Niñas del Campo y de la Ciudad \& Podestá-Siri, 2007), en el que niños de dos comunidades diferentes de Puebla, en México, colaboran horizontalmente, es decir, entre ellos, para "hablar de sus representaciones sociales sin que se establezca un diálogo directo [con la investigadora]" (Niños y niñas del Campo y la Ciudad \& Podestá-Siri, 2007, p. 29). En la investigación motivo de esta reflexión, los estudiantes trabajaron entre ellos para coinvestigar, cointerpretar y coescribir sobre el contexto en el que conviven todos los días.

Con las diferencias inherentes del presente análisis, la docente instruyó a sus estudiantes, como lo hiciera Podestá-Siri en el trabajo antes referido (Niños y Niñas del Campo y de la Ciudad \& Podestá-Siri, 2007). El curso impartido por Vilma consistió en tres unidades. En la primera se introdujo la etnografía como enfoque para el estudio de la cotidianidad educativa (Clemente \& Higgins, 2008). En la segunda unidad, los estudiantes elaboraron sus propios retratos etnográficos, con énfasis en cuestiones de identidad y locaciones sociales, como, por ejemplo, etnicidad, género y 
clase social (López-Gopar, 2016). La última unidad, la más larga del curso, consistió en el trabajo etnográfico que se describe en este artículo: la construcción del contexto de la investigación. El producto de esta etapa fue evaluado tanto por la profesora como por los propios estudiantes.

De esta manera, los siguientes apartados corresponden a la reflexión que las tres autoras hacemos sobre la narrativa de la docente, su interacción con los estudiantes y los textos producidos. Los estudiantes (a los que hemos identificado de manera colectiva como estudiantes y de manera individual con sus nombres propios) y la profesora Vilma (cuyos comentarios aparecen en cursiva) discuten los diferentes momentos en los que se planea, se lleva a cabo y se concluye la construcción del contexto de investigación.

\section{Negociando el desarrollo del trabajo etnográfico}

El trabajo colaborativo de campo se inició con una actividad dentro del salón de clases, la cual giró en torno a la definición de la pregunta de investigación. Después de una nutrida discusión y de varias propuestas, la pregunta quedó redactada de la siguiente manera: "¿Cómo es la vida social de la escuela en que convivimos diariamente?". A partir de tal interrogante, se formaron equipos, con la finalidad de seleccionar las áreas del espacio social en el que se movían cotidianamente los estudiantes: Dirección, pasillos, centro de cómputo, cafetería, biblioteca y áreas administrativas. Elena, inicia la conversación: "Hay que empezar por algún lugar, por la sala, por ejemplo..., que es la más limpia de mi casa". Es evidente que, para Elena, de su escuela, del "lugar donde se lleva a cabo la investigación", hay que mostrar lo presentable, lo que está más limpio. "Si va a ser leído por otros", parece pensar Elena, "entonces se tienen que llevar una buena impresión del lugar donde estudiamos". Esta percepción se une a la decisión implícita de los jóvenes de no tomar en cuenta los baños, a pesar de la sugerencia de su profesora. Sin embargo, más adelante, los comentarios en el aula nos permiten dilucidar que los estudiantes hacen que su percepción de la realidad se aleje de la versión oficial o tradicional de lo que se concibe como un espacio universitario:

Israel: - ¿También tenemos que decir cuando hablemos de los salones que hay cuarteaduras en los techos y no las han arreglado? [Risas.]

Elena: -iSí! Porque en la página oficial es un poco artificial, porque allí no dicen de los paros, de que el internet en los salones no funciona. La información de la página oficial es cierta, ipero no tanto! 
Cire: - Cuando ingresé acá, busqué en el internet, en la página oficial de la Facultad, y sí, te da información del lugar, de lo que vas a estudiar, pero hasta que ingresé acá me di cuenta de lo que realmente era.

Israel: - Sí, icomo que hay un contexto muerto y un contexto vivo! El muerto, pues está muerto. No cambia, y el vivo sí cambia, como cambian los estudiantes, los maestros. Creo que es frío el contexto muerto, muy numérico, como la metodología cuantitativa [risas]. iYa ven como sí sé! [Risas.]

El primer comentario de Israel lleva detrás una visión crítica sobre las condiciones materiales del contexto donde pasan varias horas de su vida diaria, una descripción sobre cómo se percibe y se experimenta la realidad. Esta idea se fortalece con lo mencionado por Elena sobre el carácter "artificial" e incluso de "cierta, pero no tanto" de la información oficial e institucional. Cire concuerda con Elena e Israel en que existe una discrepancia entre la representación promocional de la escuela en medios digitales y la que viven cotidianamente en la Facultad. Ante los comentarios de los jóvenes, y con el apoyo de Vilma para que se atrevan a decir lo que no es oficial, Israel interviene otra vez con la nominación que nos inspiró para titular este artículo: el contexto vivo. El contexto vivo de Israel es cambiante y fluido, y se opone a la verdad oficialista del contexto universitario al que se refieren los estudiantes. El contexto muerto, al contrario, es consonante a la realidad positivista, representada por la metodología cuantitativa de la investigación social. Para Israel, su facultad tiene personas que se mueven, que están vivas, y eso es lo que vale la pena representar en sus trabajos de tesis. Cuando Vilma nos platicó (a las otras dos investigadoras de este estudio) este episodio, crecieron nuestras expectativas sobre la formación etnográfica de los estudiantes, pues realmente estaban captando la realidad/representación etnográfica (Sánchez-Carretero, 2003).

Siguiendo con la narrativa de Vilma, la discusión pasa del contenido de la investigación a la forma de obtener la información. Según Salma, normalmente, los profesores, al dejar una tarea cuyo objetivo sea obtener datos para una investigación, se concretan en dar indicaciones básicas: "Normal es cuando te dicen: 'Investiga con un maestro o en la biblioteca'. Es algo muy normal, y acabas y ya". Ese "acabas y ya" parece indicarnos que lo más importante es deshacerse del trabajo, no hacerse cargo. Aquí la definición de la normalidad vuelve otra vez a lo que es aceptado, más que a lo que es aceptable. Escuchando las palabras de Salma, Vilma piensa: "La investigación cuando termina, termina. No como el contexto vivo, que 
sigue allí, cambiando, merodeando los pasillos, como el Canelo [el perro callejero adoptado por la comunidad]".

De allí, Vilma los dirige al tema que trae en la agenda, la colaboración. La visión individualista es una de las tradiciones que ha impregnado la elaboración de las tesis de grado en las universidades. En esta fórmula, los estudiantes trabajan de manera personal y la interacción más constructiva y válida es la que establecen con su asesor, pues los otros estudiantes no son reconocidos como valiosos en el proceso de conformación del trabajo de investigación. Mientras que, en la colaboración investigativa de corte etnográfico, los compañeros comparten un objetivo común y suman sus esfuerzos para alcanzar la meta (Johnson, Johnson \& Holubec, 2006). El conocimiento generado, a partir de la estructura colaborativa, en palabras de los estudiantes, se conformó con la participación de los compañeros: "Todos hablamos de cómo íbamos a investigar, discutimos bastante para hacer el trabajo, porque a veces pensábamos distinto y teníamos que llegar a un acuerdo" (Elías).

Como lo mencionan Onrubia \& Mayordomo (2015), el conflicto sociocognitivo está asociado a la colaboración, y se presenta cuando dos o más alumnos aportan puntos de vista divergentes en relación con una tarea específica. Los estudiantes, ante tal situación, ponen en marcha la revisión y reestructuración de sus propias representaciones, lo que provoca una negociación que promueve el aprendizaje no solo de conocimiento, sino también de las habilidades prosociales necesarias para vivir en comunidad (Johnson et al., 2006).

Lo anterior se puede observar con Susana y su equipo, que tuvieron una situación contingente que los llevó a detenerse y buscar una solución:

Ante la falta de un compañero, hablamos con él y volvimos entre todos a revisar el texto, ver los avances y ver qué faltaba, fue como repasar la tarea nuevamente, ver que teníamos y lo que faltaba; fue como ver claramente el panorama, discutir con los compañeros. Al final nos sorprendimos de lo mucho y bien que escribimos.

Siguiendo con el proceso de colaboración, se planteó que este se llevaría a cabo en tres niveles: coinvestigación, cointerpretación y coautoría (Huerta-Cordova et al., 2019). Con los niveles explicitados por Vilma, los estudiantes negociaron, de manera hipotética, cómo se llevaría a cabo cada una de las etapas. En la primera, la coinvestigación, los equipos decidieron que solamente un integrante del grupo realizaría la observación, pero que trabajarían en parejas las entrevistas y la toma de fotografías. Además, uno 
solo sería el entrevistador y el otro desempeñaría el rol de asistente (grabar con el teléfono, tomar algunas notas, etc.).

Alondra: -En mi equipo decidimos que la observación la hiciera uno solo, porque no es tanto de exponerse, tú puedes escribir y mirar, puede parecer que haces una tarea; en cambio, en la entrevista y en las fotos, hay que hablar con la gente, pedirle permiso para tomar las fotos, entonces es mejor ir en parejas, en las parejas te ayudas a preguntar, a escribir cosas de la entrevista, te da menos pena.

Eduardo: - Para la entrevista decidimos hacer unas preguntas, cada quien decía una pregunta, unos dijeron preguntas como "¿En qué semestre vas?", básicas, por decirlo, y después, conforme avanzamos, preguntamos otras cosas como "¿Te parece que la atención de las secretarias es amable?".

Desde el momento de la planeación es posible notar una resistencia a la investigación colaborativa, pues, por ejemplo, las tareas asignadas entre los participantes son más bien actividades adjetivas a la investigación (acompañar al investigador, sostener la grabadora, etc.).

Con respecto a la cointerpretación, los estudiantes opinaron que se debían reunir todos los datos y, en una mesa de trabajo, intentar, primeramente, dar un orden a la información, una especie de categorización de los datos: "Que cada quien traiga lo que le tocó y entonces organizamos. Yo digo que al que le tocó observar sea más responsable del análisis de esa información. Claro, vamos a ayudar, pero él sabe más" (Salma). Desde su punto de vista, cada estudiante sería mayormente responsable de su actividad y de la información generada, se convertiría en una especie de experto, lo que llevaría a regresar a una situación de trabajo individual, que se opondría a la colaboración etnográfica.

Finalmente, al discutir la coautoría, los estudiantes concluyeron que sería un proceso complejo, pues debían llegar a un acuerdo sobre cómo escribir el documento final:

Eduardo: - Acá va a estar complicado, porque no tenemos muchas experiencias de hacer algo así. Tenemos que ver cómo lo hacemos. Una idea es que empiece uno de nosotros y después vemos cómo lo escribió y lo corregimos. ¿Qué piensan?

Alondra: - Sí. Puede ser una forma, pero no me convence, porque lo mejor es que todos nos involucremos, pero no sé cómo, mejor avanzamos y ya vemos a la mera hora cómo lo resolvemos. 
Además de lo expresado en las conversaciones anteriores, se abrió un espacio en donde ellos cuestionaron la colaboración. Surgieron quejas y opiniones negativas. La colaboración etnográfica se relacionó inmediatamente con las dinámicas educativas de "trabajo en equipo", de las cuales la mayoría de estudiantes mexicanos (desde el nivel preescolar hasta el universitario) tienen experiencias poco gratificantes. A pesar de que los estudiantes nunca habían trabajado la etnografía en colaboración, la mayoría opinó que trabajar colaborativamente "no les gusta", "saca de onda", "el trabajo no resulta equitativo" y "surgen conflictos".

Carlos inclusive propuso una forma democrática para resolver el asunto ("Yo voto porque sea individual"). Mover la situación a un nivel político de votación nos sonó (a las otras dos investigadoras) interesante, pero Vilma decidió ignorar la propuesta. Ella pensó que esta pequeña imposición daría más rendimiento que el individualismo positivista al que están acostumbrados los estudiantes. Vilma los convenció de los beneficios que se ganan en la colaboración, con varias alusiones a lo educativo y demás argumentos relacionados con la cultura y la diversidad. Sin duda, el modelo negociador (Canagarajah, 1999) le da resultados y alimenta sus esperanzas: "Es el pretexto para encontrarnos, para hacernos visibles en el radar de los otros, una magnífica oportunidad para el encuentro", piensa Vilma.

\section{Iniciándose en la etnografía}

Una vez habiendo acordado la pregunta de investigación y la manera en que se trabajaría, se inició el trabajo de campo. Para empezar, se recomendó a los estudiantes, antes de iniciar la investigación que describieran los lugares que integrarían el contexto de la investigación. La finalidad de esta tarea fue que los jóvenes investigadores tomaran conciencia de las representaciones a priori que guardaban sobre el lugar de indagación antes de entrar al campo, concepciones, probablemente, nunca verbalizadas formalmente: "De repente, la tarea me sacó de onda, había que describir el lugar en donde estás mucho tiempo, primero tuve que pensar un poco, después me di cuenta que pongo poca atención a cómo es la escuela" (Emi).

La actividad los tomó por sorpresa, les demandó tiempo poder concentrarse en esos aspectos cotidianos que pasan desapercibidos, que forman parte de la rutina, y que muchas veces se obvian. Ya conscientes de sus propias formas de visualizar su escuela, los estudiantes salieron al campo a observar. Alondra comentó sobre su forma de abordar la actividad:

Bueno, yo, la verdad, me dije: "Vas y te sientas en la dirección y ves cómo está el lugar, qué cosas tienes a la vista y las cosas que casi no se ven, como una hoja con muchos números que está pegada en la pared y que la secretaria va tachando. 
Otro estudiante, Esteban, añadió:

Pues yo me fui a sentar a la oficina de la Dirección con mi cuaderno. Primero, me preguntaban si necesitaba algo. Le dije que no, que estaba haciendo una tarea. Después, venía otro coordinador y me preguntaba si necesitaba algo, y volvía a decir que no, y así varias veces. Creo que me veían sospechoso. Entonces que le habló a mi compañera y que le digo: "Vente y me ayudas a tomar las notas". Bueno, más bien yo escribía y ella me decía cosas como: "Ya pusiste que hay una mesa en el centro". Me sentí mejor con ella.

A pesar de que el grupo había decidido observar de manera individual, Esteban tuvo la necesidad de solicitar la colaboración de su compañera. A Esteban le causó sorpresa que el personal que allí laboraba no entendiera su rol como investigador.

Con respecto a la toma de fotografías, los estudiantes tuvieron sus propios criterios. Uno de ellos fue mostrar una imagen fotográfica del espacio físico estudiado y, el otro, de un corte más íntimo, fotografiar a las personas que daban información:

Primero, tomé fotografías de las oficinas. La idea fue que hubiera una foto de lo que describíamos, pero cuando fuimos a la cafetería y entrevistamos a las personas, les pedimos permiso para tomarles una fotografía, porque se nos hizo más bonito, más cercano. Era importante que las personas aparecieran, como don Manuel (Eduardo).

En cuanto a las entrevistas, los estudiantes, organizados en grupos de dos o tres, habían escrito un guion de preguntas; sin embargo, tuvieron que dejar la guía de entrevista a un lado, debido a que esto les dificultó hacer la tarea de entrevistar. En concreto, les impidió un contacto más directo con la persona entrevistada y físicamente les limitó la movilidad: "Mi compañero me dijo: 'Dame ese guion que estorba'. Y la verdad sí estorbaba, hasta para moverse estorbaba. Al final, concluimos que con el guion la entrevista era muy cuadrada, sin el guion fue más sencillo" (Ana).

Otro de sus comentarios fue que salieron al campo con un limitado criterio para seleccionar a los entrevistados. Ya en el campo, fue la oportunidad y la disposición de las personas para dar información lo que definió quién sería entrevistado.

Eduardo: - Salimos a ver quién estaba en el lugar, y veíamos si nos podía decir algo. 
Alondra: -Ajá, y si quería comentar, aprovechábamos, y si no, buscábamos a otro. Hasta nos fijábamos en sus caras, para ver quién se veía buena gente y aceptaba la entrevista.

Una vez realizado el trabajo de campo, se elaboraron las transcripciones de entrevistas y los borradores de observaciones. Los datos estaban sobre la mesa. El análisis, aunque limitado, comenzó a desarrollarse. Como parte del análisis de los textos iniciales (en donde describen ellos mismos sus concepciones de los lugares), los estudiantes decidieron encontrar las coincidencias y las diferencias en la información recolectada:

Salma: - Yo digo que debemos empezar por los textos personales. Hay que ver los textos que escribió cada uno y las cosas en las que estamos de acuerdo, y después las cosas particulares.

José: —Pues, todos estuvimos de acuerdo que la oficina de la maestra Kiara tiene un escritorio, tres sillas y que allí se resuelven cosas de las inscripciones. Lo diferente es que Zully dijo que había muchos recuerditos, y que están llenos de polvo y que no hay mucho espacio para los papeles.

Israel: — ¿Y ahora cómo vamos a hacer este trabajo?

Zully: - Yo opino que leamos todo primero y que cada quien haga observaciones, y de allí vamos viendo cómo organizar.

Los estudiantes encontraron dos momentos de colaboración. El primero, en el campo, fue dividir los espacios de estudio, y el segundo fue hacer un análisis conjunto de la información, lo que Johnson et al. (2006) llaman interdependencia positiva.

\section{Visibilizando a los invisibles}

En relación con el producto escrito que se debería de entregar, los estudiantes tomaron decisiones sobre la información que incluirían en sus reportes y la que se quedaría fuera. Para ellos, por ejemplo, fue importante el descubrimiento de Salma: "Lo que más me impresionó fue que descubrimos que hay personas que son parte de la escuela y que no las tomamos en cuenta, como don Manuel o Carlos. Son como invisibles para nosotros". Se trata de los "invisibles", aquellos miembros de la comunidad que generalmente no son tomados en cuenta como "parte de la Facultad", porque, aunque proporcionan servicios, como hacer el café, estos no están relacionados con su educación. Para cambiar esta situación, los estudiantes deciden escribir sobre esa parte de la comunidad, quienes "también tienen historias que contar". 
Don Manuel es un hombre que tiene en alto el valor de la amistad, y en honor a ese bonito recuerdo de la frase de su buen amigo decidió nombrar así a su negocio. [...] Entre las varias preguntas que se le realizaron a don Manuel, hubo una que abordaba el tema de Canelo - el famoso perro de la Facultad, que se ha dado a conocer a lo largo y ancho de toda la Universidad gracias a sus repentinos estados de humor-y también sobre lo que implicaba estar al mando del establecimiento. Y esto fue lo que nos platicó:

Don Manuel: -iClaro que conozco a Canelo! Desde que nosotros Ilegamos aquí, él ya estaba, pero no era cachorro. De hecho, dicen que está aquí desde que se construyó este edificio, y sí, Canelo ya es un perro viejo, yo le calculo que ha de tener unos 18 años... Nunca me ha atacado, de hecho me cuidaba en las noches cuando me quedaba aquí o cuando salía de plano muy tarde. Fíjate que a veces ocurrían cosas extrañas en la cocina, de repente se oían ruidos adentro que sí me espantaban. Pero con el tiempo eso dejó de pasar. Ya sabes, a lo largo de cinco años uno ha visto muchas cosas y también hemos sido cómplices de varias cosas, sobre todo con los muchachos, que de pronto nos piden que les guardemos cosas, que entreguemos regalos para la dama, que el chocolatito, que un postrecito, y pues así es este trabajo, de estar conociendo y tratando a la gente, porque pues con tanto tiempo uno logra aprender mucho de las personas, sobre todo de los muchachos, y pues aquí estamos tratando de responder, aunque pues sí, muchas veces no se puede, y siempre hay personas que se desesperan, ¿pero qué puedo hacer? Aquí la comida se prepara al instante y hay que tardarse un poquito, pero pues es cuestión de cada quien, nosotros hacemos lo mejor que podemos para brindar el mejor servicio (extracto de la tesis del equipo de Julián, 2018).

La idea de escribir el contexto vivo fue proporcionar una representación alejada de la "oficial", más cercana a lo que los estudiantes viven de manera cotidiana. Es evidente que el hecho de escribir sobre estos personajes los hace pertenecer, los incluye, los visibiliza. Después de producidos, los textos hacen más que comunicar, se vuelven performativos (Austin, 1976). Los textos no solo significan, sino que también hacen; no solo reflejan realidades, sino que también las construyen. En este sentido, con su escritura, los estudiantes otorgaron la membresía a los dependientes de los puestos de café y comida, y con esto reconstruyeron la realidad de la facultad en la que estudian.

En este análisis de la escritura y sus procesos, es importante hablar de otro tipo de invisibilidad: lo que los estudiantes decidieron no escribir. Las historias no contadas son esos episodios vistos en las observaciones y 
escuchados en las entrevistas que los estudiantes no incluyeron. Estos elementos nos remiten a dos situaciones que merecen ser analizadas.

Por un lado, Zaira decide no escribir acerca de las historias de los pasillos. "[Un asunto] que me sorprendió es el de los pasillos de la Facultad, pareciera que no tienen mucha relevancia, pero si esos pasillos hablaran, cuántas historias no contarían". Isidro también decide omitir el hecho de que hay estudiantes que van a la biblioteca a dormirse: "Mientras esperaba a que abrieran la biblioteca, unos chavos estaban platicando que van a la biblioteca a dormirse".

Zully, por su parte, deja fuera el comentario negativo sobre el acceso restringido a los sanitarios. Para los tres, estos episodios son cuestiones que "parece no tienen relevancia", ni son suficientemente importantes para incluir en el contexto de la tesis. Decididamente no son elementos que hayan visto relatados en contextos de tesis "normales", según la expresión de Salma. Relacionado con este sentir, está el hecho de que lo social, que parece que se opone a lo académico, no es motivo de análisis dentro del tipo de trabajo que se pide en una carrera universitaria, aunque el cuestionamiento de esa información pueda arrojar luz sobre las condiciones sociomateriales (ipor qué los estudiantes se van a dormir a la biblioteca?) de los actores principales del contexto que se está investigando. De acuerdo con Héctor, "esa información no estamos acostumbrados a ponerla en los trabajos que son bien formales", y completa su intervención con un toque multicultural: "Este trabajo es de otro tipo... of a different nature...".

\section{Considerando al lector implícito}

La escritura académica como requerimiento de un curso escolar generalmente tiene una estructura social vertical. Es decir, el lector es, sin duda alguna, el profesor que asignó la escritura como tarea. Sin embargo, en el estudio en cuestión, los estudiantes tuvieron que considerar varios niveles de audiencias. El primero, el más inmediato, fue propiciado por la colaboración entre ellos, que, además, implicaba la colaboración de sus interlocutores entrevistados, lo que reveló una serie de problemáticas debido a la dificultad de obtener la información necesaria: "Fuimos a preguntar y la gente no quiso darnos las entrevistas. Dicen que no pueden, no tienen tiempo, está difícil que alguien diga sí a la primera".

Sobre la colaboración entre compañeros de grupo, los estudiantes no comentaron nada acerca de cómo influyó en su escritura saber que sus textos iban a ser leídos por sus compañeros (otros estudiantes investigadores). No obstante, podemos suponer que, debido al conocimiento que tienen de los demás integrantes del grupo, estaban pendientes de quiénes leerían y cómo leerían sus textos. La segunda audiencia, su profesora, fue definitiva 
para escribir un texto que sería calificado con estándares "académicos", "escolares", "de investigación".

El tercer nivel de audiencia, considerado por los estudiantes investigadores, fue el de los revisores y jurados de la tesis. Aquí hubo una notable preocupación, pues la mayoría expresó que la investigación que proponía la profesora no parecía tener los estándares requeridos para una tesis. No se parecía a otros textos sobre contextos en las tesis que conocieran, además de que no era posible considerar un texto colaborativo como parte de una tesis individual: "Yo voto porque sea individual, además, una tesis es individual".

Las ideas y las intenciones de los autores se ven considerablemente influenciadas por las expectativas, normas y valores de la audiencia y de la comunidad, pero, aunque dudan, el resultado les hace estar orgullosos ("Nos quedó muy profesional", "Nos gustó", "Nos quedó chido el trabajo"). Sus escritos equivalen a un constructo mediado por la lucha, la colaboración

y la interacción entre el escritor, el lector y la comunidad de pensamiento (Canagarajah, 2002).

La propuesta para que los estudiantes se iniciaran en la investigación etnográfica ("Ahora vamos a construir el contexto de otra manera, etnográficamente, como lo hicimos con sus autobiografías") definitivamente tuvo una reacción abierta por parte de ellos, quienes expresaron que no estaban acostumbrados a escribir un texto sin consultar otros textos "oficiales" para legitimar su escritura, esa convención académica de necesitar que otros escritores validen lo que dicen los menos expertos. Asimismo, en cuanto a la forma, algunos estudiantes refirieron estar satisfechos con la presentación del texto: "Se ve muy profesional", "Nos quedó chido". Otros hablaron de haber revisado elementos formales, como "errores ortográficos, falta de formato y cohesión...".

\section{Reflexionando sobre las condiciones materiales}

Vilma en sus propias notas escribió sobre la mala condición de las computadoras del grupo: "Los muchachos buscan los enchufes porque las pilas ya no funcionan. Sus computadoras, pesadas y antiguas, no son de última generación, recordatorio de la condición socioeconómica de mis estudiantes". Esto nos hizo reflexionar sobre las condiciones materiales de los investigadores y sus informantes.

De entrada, tuvimos que admitir que habíamos asumido que todos los estudiantes tenían el mismo acceso a los recursos materiales necesarios para producir textos. Teníamos que reconocer que, cuando asignamos una tarea, no tomamos en cuenta que no todos los estudiantes tenían la posibilidad de llevar computadoras portátiles a la escuela; es más, algunos 
no tenían computadoras en casa. Al considerar la dimensión material de la escritura, corresponde, también, considerar que, por lo general, estos estudiantes, que carecen de facilidades de conectividad y de medios electrónicos de escritura, son los que tienen que trabajar para subsistir y para compensar la falta de recursos. Por ende, son ellos los que cuentan con menos tiempo para dedicar a la escritura.

Aquí no se trata de poner al estudiante investigador en posición de déficit, sino de aceptar las condiciones y diferencias en las que se mueven estos escritores. No se puede obviar el hecho de que la escritura implica el acceso a recursos materiales para componer los pensamientos. La noción de que solo la pluma y el papel es lo que necesita un buen escritor es demasiado romántica en estos tiempos de la educación, inclusive en esta universidad pública tercermundista. A los buenos escritores también los determina su capital económico y cultural (este último íntimamente relacionado con el primero).

\section{Conclusiones}

La escritura es más que lenguaje y estructura. Es la representación de la realidad: una encarnación de valores y una presentación de sí mismo. La misma forma está basada en diversas convenciones de textualidad, valores de apropiación y actitudes sobre el estilo ("escribimos muchas páginas. También eso me gustó, el trabajo también se ve profesional"). Ya que la escritura se trata no solo de reglas, sino de cómo usarlas - es decir, con que propósito y actitud - , entonces, se trata de un área polémica de diferencias culturales y preferencias ideológicas (Canagarajah, 2002). Aquí vuelve a tener relevancia la inclinación de los estudiantes de reflejar en su escritura un contexto vivo, diferente al contexto que ha sido construido por las autoridades universitarias.

El contexto vivo es definido así por Israel: "El contexto muerto... no cambia, y el vivo sí cambia, como cambian los estudiantes, los maestros". Es donde se habla de las condiciones en las que se usan los edificios después del terremoto de 2017 ("cuando hablemos de los salones que hay cuarteaduras en los techos y no las han arreglado"), un contexto vivo en donde se hable de que los estudiantes se duermen en la biblioteca y donde hay perros callejeros adoptados por gente ya no tan "invisible".

Un contexto vivo en donde se considera que los valores que están implícitos en la forma y en la norma textual se pueden modificar para representar valores alternativos (Canagarajah, 2002). Decididamente, los procesos y los productos de escritura en este estudio no son perfectos, pues los procesos de interpretación etnográfica son incipientes. Sin embargo, se 
logró ir más allá de las convenciones institucionales sobre la manera de interpretar el contexto educativo y el contexto de interacción cotidiana.

Los procesos de escritura de los estudiantes quedaron documentados durante el curso del estudio, la colaboración entre ellos hizo evidente estos procesos, que fueron acompañados por las correspondientes problemáticas sociales comentadas anteriormente. Los procesos de escritura se desarrollaron a través del tiempo; hubo muchos comienzos fallidos y muchas más revisiones para comunicar lo que los escritores querían decir, y ciertamente hubo complicaciones merodeando este proceso (Canagarajah, 2002). Y, lo que es más sobresaliente, los textos siguen siendo decodificados e interpretados ahora mismo, que estamos escribiendo sobre ellos, y en el futuro, cuando las tesis se defiendan y este manuscrito se publique. La escritura en su dimensión histórica no es un producto sino un proceso.

A partir de lo discutido en los apartados anteriores, las autoras, al reflexionar sobre el proceso y producto de la investigación llevada a cabo por los estudiantes, coincidimos en cómo este tipo de ejercicios educativos y etnográficos contribuyen a seguir debatiendo la perspectiva epistemológica y política de la propia etnografía. Además, hemos tenido la oportunidad de reflexionar sobre las contribuciones a los procesos de aprendizaje colaborativo con jóvenes en formación como profesores de lenguas y las dificultades de construir un objeto de estudio y de iniciarse como etnógrafos.

En la colaboración, el conocimiento se construye de manera colectiva a partir de la estructuración y supervisión que el profesor hace de la tarea y, especialmente, de la negociación que se establece entre los estudiantes, lo que, en palabras de Onrubia \& Mayordomo (2015), tiene que ver con la perspectiva sociocultural del aprendizaje, en donde se establece una coconstrucción colaborativa de ideas y significados entre las personas. En la coconstrucción, los alumnos intercambian ideas o ayudas y producen nuevas ideas, a partir de la revisión, discusión de las aportaciones de cada uno.

Una de las primeras conclusiones es que este trabajo etnográfico colaborativo, por más básico que haya sido, hizo posible el cambio de las condiciones de las situaciones de poder clásicas en los ambientes escolares y universitarios. En este caso hemos visto cómo cambió la dinámica en cuanto a la posición de la profesora en una situación educativa vertical, balanceándola con la interacción horizontal entre estudiantes para la generación del conocimiento. Aunque no podemos afirmar que el trabajo colaborativo etnográfico haya sido la raíz del cambio, sí estamos seguras de que incidió en los resultados.

El darse cuenta de que los documentos públicos que supuestamente describen el contexto de la institución no concuerdan con el día a día de los estudiantes dio pie a que se generaran escritos sobre su escuela, utilizando 
su observación e interacción en ella. Para nosotras son evidentes las posibilidades que abre el hecho de proponer un cambio en la redacción del contexto a partir de una investigación en la que este permita entender dinámicas sociales que pocas veces se toman en cuenta.

Una de las potencialidades del trabajo etnográfico fue la manera colectiva en la que se observó, registró y construyó el conocimiento sobre el contexto social (Corona-Berkin \& Kaltmeir, 2012). Este proceso también permite reflexionar sobre cómo existe resistencia de los estudiantes, pero, también, de otros actores, para colaborar en la investigación. Asimismo, la experiencia les permitió superar algunas barreras sociales, comunicativas y culturales en el ámbito educativo. De esta manera, utilizar este tipo de procesos investigativos - en programas de licenciatura distintos a la antropología - propicia la obtención de resultados educativos y de investigación cualitativa de características más reflexivas y dialógicas (Dietz, 2012).

En la experiencia de formación de nuevos etnógrafos, las autoras tratamos de crear las condiciones para que los estudiantes tuvieran a la mano herramientas y habilidades para ingresar al dato, a la forma básica del conocimiento empírico y etnográfico, para la construcción del contexto de investigación, el cual implicó distintas controversias dentro del proceso de colaboración entre pares.

Si bien, a los estudiantes, este ejercicio reflexivo les podrá tomar mayor tiempo de práctica, en el proceso de colaboración del presente artículo de reflexión, las autoras decidimos explorar dicho proceso desde la parte textual: por un lado, al evidenciar el dato (qué sucedió al estar allí) y, por el otro, al construir sentido etnográfico (la visibilización de las voces) para la interpretación del sujeto de estudio, los silencios y su interrelación en el momento de encarar el proceso de escritura etnográfica (Sánchez-Carretero, 2003).

Para finalizar, dentro del análisis de los procesos de escritura, nos pudimos dar cuenta de que la diferencia económica de los estudiantes trae consigo niveles de ventaja para unos. Es simple, se necesita cierto nivel de privilegio para contar con el tiempo necesario para escribir. Escribir bien no depende nada más de las habilidades cognitivas, sino, también, de los recursos materiales disponibles. Desgraciadamente, tenemos que admitirlo, en la presente investigación no ahondamos en estos factores. Pasamos por alto comentarios, orales o escritos, que pudieran llevar detrás un fondo de condición social.

Nunca nos preguntamos, ni tampoco se preguntó a los estudiantes cuestiones como las siguientes: ¿por qué no todos los estudiantes traen portátiles a la escuela?, ¿por qué algunos estudiantes utilizan la biblioteca para dormir?, ¿qué hubo detrás de la ineficacia del estudiante que "no traía 
consigo de manera digital su investigación en USB"? ¿fue falta de compromiso o falta de tiempo la razón por la que "el compañero no envió su trabajo completo, [y], por ende, [no pudieron] completar bien esa parte"? Tendremos que esperar a una futura investigación para ahondar en la dimensión material de la escritura etnográfica en este contexto educativo.

\section{Sobre las autoras}

Vilma Huerta-Cordova es doctora en Estudios Críticos del Lenguaje y profesora e investigadora en la Facultad de Idiomas de la Universidad Autónoma Benito Juárez de Oaxaca, México, en los programa educativos Licenciatura en la Enseñanza de Idiomas y Maestría en Enseñanza Crítica de Lenguas. Forma parte del Cuerpo Académico en Lingüística Aplicada Crítica de la Facultad de Idiomas, y sus áreas de interés son el aprendizaje colaborativo, la tutoría entre iguales y las relaciones interpersonales en el aula.

Ángeles Clemente-Olmos tiene un doctorado en Lingüística Aplicada de la Universidad de Londres, Inglaterra. Sus intereses académicos son el estudio etnográfico colaborativo de la construcción, deconstrucción y reconstrucción de identidades de niños y jóvenes de comunidades subalternas a través de sus actividades lingüísticas y culturales.

Lorena Córdova-Hernández es doctora en Antropología. Profesora-investigadora de la Universidad Autónoma Benito Juárez de Oaxaca, México. Miembro del Sistema Nacional de Investigadores (Conacyt) y del Cuerpo Académico Lingüística Aplicada Crítica, de la Facultad de Idiomas de la Universidad Autónoma Benito Juárez de Oaxaca, México. Sus líneas de investigación-colaboración comunitaria son las políticas públicas de cultura, la revitalización de lenguas y la planificación lingüística.

\section{Referencias}

Austin, J. L. (1976). How to Do Things with Words. Oxford: OUP.

Canagarajah, S. (1999). Resisiting Linguistic Imperialism in English Teaching. Oxford: Oxford University Press.

Canagarajah, S. (2002). Critical Academic Writing and Multilingual Students. Michigan: The University of Michigan Press.

Clemente A. \& Higgins, M. J. (2008). Performing English with a Postcolonial Accent: Ethnographic Portraits from Oaxaca, Mexico. Londres: The Tufnell.

Corona-Berkin, S. \& Kaltmeier, O. (2012). Introducción. En S. Corona-Berkin \& O. Kaltmeier (Eds.), En diálogo: metodologías horizontales en ciencias sociales (pp. 11-21). Barcelona: Gedisa.

Dietz, G. (2012). Reflexividad y diálogo en etnografía colaborativa: el acompañamiento etnográfico de una institución educativa "intercultural" mexicana. Revista de Antropología Social, 21, 63-91. doi: 10.5209/rev_RASO.2012. v21.40050 
Domínguez-Gaona, M., Crhová, J. \& Molina-Landeros, R. (2015). La investigación colaborativa: las creencias de los docentes de lenguas. Revista Iberoamericana de Educación Superior, 6(17), 119-134. Recuperado de https://www. redalyc.org/pdf/2991/299141540007.pdf

Geertz, C. (1973). The Interpretation of Culture. Nueva York: Basic.

Guerrero, A. L. (2018) Collaborative ethnography as a possibility of developing visible and constructive agency with children in displacement conditions in Colombia. Acta Paedagogica Vilnensia, 39, 31-44.

Heath, S. B. (1984). Ways with Words: Language, Life and Work in Communities and Classrooms. Cambridge, UK: CUP.

Huerta-Cordova, V., Clemente, A. \& Córdova-Hernández, L. (2019). Etnografía colaborativa: somero estado de la cuestión de los trabajos sobre y con niños/as y jóvenes en México. Interlocución, producción de conocimiento y coautoría. Revista del Cisen Tramas/Maepova, 7(2), 247-280. Recuperado de http://ppct.caicyt.gov.ar/index.php/cisen/article/view/14608

Johnson, D., Johnson, R. \& Holubec, E. (2006). El aprendizaje cooperativo en el aula. (G. Vitale, Trad.). Buenos Aires: Paidós.

López-Gopar, M. (Coord.). (2016) Historias de vida de estudiantes universitarios de origen indígena. Oaxaca: UABJO.

Milstein, D., Clemente, A., Dantas-Whitney, M., Guerrero A. L. \& Higgins, M. (2011).Encuentros etnograficos con niñ@s y adolescente. Entre tiempos y espacios compartidos. Buenos Aires: Miño y Dávila.

Niños y Niñas del Campo y de la Ciudad \& Podestá-Siri, R. (2007). Encuentro de miradas. El territorio visto por diversos autores. México, D. F.: Secretaría de Educación Pública y Coordinación General de Educación Intercultural Bilingüe.

Onrubia, J. \& Mayordomo, R. (2015). El aprendizaje cooperativo elementos conceptuales. En R. Mayordomo \& J. Onrubia (Coords.), El aprendizaje cooperativo (pp. 179-651). Barcelona: OUC.

Rappaport, J. (2007). Más allá de la escritura. La epistemología de la etnografía en colaboración. Revista Colombiana de Antropología, 43, 197-229. Recuperado de http://www.humanas.unal.edu.co/colantropos/files/9114/ 7414/9089/Mas_alla_de_la_escritura_Rappaport.pdf

Roberts, C., Byram M., Barro A., Jordan, A. \& Street, B. (2001). Language Learners as Ethnographers. Clevedon: Multilingual Matters.

Sánchez-Carretero, C. (2003). Voces y escritura: la reflexividad en el texto etnográfico. Revista de Dialectología y Tradiciones Populares, 58(1), 71-84. Recuperado de https://doi.org/10.3989/rdtp.2003.v58.i1.164

Woods, P. (1993) Researching the Art of Teaching: Ethnography for Educational Use. Londres: Routledge. 